\title{
Does Dopamine Agonist Hormonal Therapy for Macro-Prolactinoma Affect Future Surgery?
}

\author{
Mohamed Raafat Kandeel, Magdy A. Elhawary, Osama Elghannam \\ Department of Neurosurgery, Faculty of Medicine, Al-Azhar University, Cairo, Egypt \\ Correspondence author: Mohamed Raafat Kandeel, Phone: +20 01001129203 - Email:kandeelneurosurgery@gmail.com
}

\begin{abstract}
:
Background: Controversy regarding first line therapy for macro-prolactinoma patients had been of debate for many decades. Several reports suggested that dopaminergic agents (DAs) medications are accused for prolactinoma fibrosis. Whether fibrosis has positive or negative influence on the management of prolactinomas is still unclear.

Aim of the work: was to compare the intraoperative tumor consistency, intraoperative difficulties and complications both pre- and post-operative between macro-prolactinoma patients who received DA medications and who did not receive prior to surgery.

Patients and Methods: Twenty-five macro-prolactinoma patients who underwent primary pituitary surgery, sublabial trans-sphenoidal microscopic removal, of macro-prolactinoma grouped into two groups. Group 1 included patients who were treated by DA medication, combined therapy with Bromocreptine and Cabergoline, prior to surgery for at least 3 months. Group 2 included patients who didn't receive any preoperative DA medications. We compared the intra-operative texture of the tumor, intraoperative and post-operative complications together with the extent of surgical excision and hormonal remission achieved.

Results: No significant statistical difference as regards intra-operative tumor consistency in relation to preoperative hormonal treatment. The rate of intra-operative and post-operative complications has no relation to the pre-operative hormonal therapy by DAs nor to the intraoperative tumor consistency. The only factor that affect the extent of surgical excision is the pre-operative tumor size graded by KNOSP classification and this extent of surgical excision has direct implication on the post-operative hormonal remission rate.

Conclusion: It could be concluded that there is no risk of more tumor fibrosis nor increase in the complication rate due to DA medication prior to surgery. So, using dopamine agonist as the primary treatment for prolactinoma while Surgical excision is deferred for cases of failure or intolerance of side effects of the the hormonal therapy. Key words: Pituitary adenoma, Macroprolactinoma, Dopamine agonists, Bromocreptine, Cabergoline.
\end{abstract}

\section{Introduction:}

Controversy regarding first line therapy for macro-prolactinoma patients had been of debate for many decades. Prior to the discovery of dopaminergic agents (DAs), these tumors were treated surgically ${ }^{(\mathbf{1})}$. DAs, due to their clinical effectiveness and low toxicity profile, have become the first line treatment, reserving surgery for patients who failed medical therapy or for neurosurgical emergencies such as apoplexy or neurologic deficit involving the visual pathways ${ }^{(2,3)}$.

It has been reported that prolactinomas exposed to BROM may develop tumor fibrosis ${ }^{(2-10)}$, which has generated a renewed interest in surgical treatment of prolactinomas, and has promoted some controversy regarding first line therapy ${ }^{(11-14)}$.

Several reports suggested that DA medications are accused for prolactinoma fibrosis. Bevan and others $^{(2,5,10)}$ have reported that bromocreptine is associated with histological perivascular tumor fibrosis, but whether fibrosis has positive or negative influence on the management of prolactinomas is still of debate. as studied by Molitch and others ${ }^{(11-14)}$.

We hypothesized that there is no correlation between the intraoperative tumor consistency, occurrence of intraoperative and post-operative complications in relation to DA medication use before surgery.

The objective of the current study was to compare the intraoperative tumor consistency, intraoperative difficulties and complications both pre- and postoperative between macro-prolactinoma patients who received DA medications and who did not receive prior to surgery.

\section{Patients and Methods:}

This retrospective comparative study included a total of 25 patients (17 males, 8 females) who underwent primary pituitary surgery, sub-labial trans-sphenoidal microscopic removal, of macroprolactinoma, attending at Department of Neurosurgery "Neurosurgery Gama and Cobalt center". Approval of the ethical committee and a 
written informed consent from all the subjects were obtained. This study was conducted between January 2016 and December 2017.

\section{Patient inclusion and exclusion criteria:}

Patients had been selected regardless of their age group and sex. Chosen patients had MRI brain demonstrating a macro-prolactinoma $>10 \mathrm{~mm}$ in diameter. We had excluded from this study any patient who had previous pituitary surgery, cranial radiation therapy or complaining of medical problems that would increase the surgical complications by its nature. The study was approved by the Ethics Board of Al-Azhar University.

The patients had been distributed between two groups. Group 1: included 12 patients (48\%) and it comprehended the patients who were treated by DA medication, combined therapy with Bromocriptine and Cabergoline, prior to surgery for at least 3 months with no response, little response and/or intolerance to side effects of DAs. Group 2: included patients who didn't receive any preoperative DA medications and prefer to go for surgery from the start on their own will or due to severity of their presenting symptoms $52 \%(\mathrm{n}=13)$.

\section{Preoperative assessment:}

Preoperative assessment done for all the included patients with MRI brain with and without contrast, serum prolactin level, visual field testing and preoperative investigations according to ASA.

\section{Intraoperative:}

A sub-labial trans-sphenoidal microsurgical excision of macro-prolactinoma was done by the same surgeon for the all 25 patients. PENTERO 900 microscope had been used while the patients were in a supine position with maximum head extension and head fixation by myefield.

We compared the intra-operative texture of the tumor as described by the operating Neurosurgeon in the operative notes. The intraoperative and postoperative complications had been recorded. postoperative MRI pituitary with IV contrast was done on the next two days following the surgery.

\section{Histopathological assessment:}

Histopathological diagnosis was based on conventional light microscopy and specific immunohistochemistry detecting prolactin in accordance with the WHO classification of tumors of the endocrine system ${ }^{(15,16)}$.

\section{Postoperative follow up:}

A postoperative clinical, neuro-radiological and biochemical evaluation was performed at 4-6 weeks by the operating neurosurgeon. MRI brain with and without contrast, serum prolactin and Visual field were routinely done at 6 weeks follow up.

\section{Statistical analysis:}

Statistical analysis was done using IBM@ SPSS $\odot$ Statistics version 22 (IBM $\odot$ Corp., Armonk, NY, USA). Numerical data were expressed as mean and standard deviation or median and range as appropriate. Qualitative data were expressed as frequency and percentage. Chi-square test or Fisher's exact test was used to examine the relation between qualitative variables. For not normally distributed quantitative data, comparison between two groups was done using Mann-Whitney test (nonparametric t-test). All tests were two-tailed. A pvalue $<0.05$ was considered significant.

\section{Results: \\ Patients characteristics:}

The mean age of the studied patients was $42 \pm 14$ years while the median was 41 years (range from 19 to 74 years). Forty-four percent of patients $(n=11)$ were $<40$ years at presentation, while $56 \%$ of them $(n=14)$ were $\geq 40$ years. Male to female ratio was 2.1:1.

Group 1 includes 12 patients (48\%) who were treated by DA medication prior to surgery for at least 3 months. Group 2 includes 13 patients (52\%) who didn't receive any preoperative DA medications.

The number of females who received preoperative hormonal therapy were higher than males $(62.5 \%$ vs $41.2 \%$; $\mathrm{P}=0.411)$. as regards the age group, we found no statistical difference in patients $\geq 40$ years ( $50 \%$ for each). A very small difference in the age group $<40$ years $(54.5 \%$ Group 1 vs $45.5 \%$ Group $2 ; \mathrm{P}=0.821$ ).

Group 1 patients were found to be inversely proportionate to Knosp tumor classification of prolactinoma. with the highest percentage of patients $66.7 \%$ of patients with Knosp grade 2 tumors preferred to receive hormonal therapy first. While $44.4 \%$ of Knosp grade 3 and only $28.6 \%$ of Knosp grade 4 tumors did receive hormonal therapy prior to surgery (66.7\% Knosp 2, 44.4\% Knosp 3, 28.6\% Knosp 4; $\mathrm{P}=0.378)$. All these differences when analyzed were found statistically insignificant (table 1).

Table 1: Hormonal treatment in relation to demography and pathology 


\begin{tabular}{|c|c|c|c|c|}
\hline & & 1 & 2 & \\
\hline \multirow{2}{*}{ Sex } & Male (17) & $10(58.8 \%)$ & $7(41.2 \%)$ & \multirow{2}{*}{$\mathrm{P}=0.411$} \\
\hline & Female (8) & $3(37.5 \%)$ & $5(62.5 \%)$ & \\
\hline \multirow{2}{*}{ Age } & $<40$ years $(11)$ & $6(54.4 \%)$ & $5(45.5 \%)$ & \multirow{2}{*}{$\mathrm{P}=0.821$} \\
\hline & $\geq 40$ years $(14)$ & $7(50 \%)$ & $7(50 \%)$ & \\
\hline \multirow{4}{*}{ Knosp Classification } & Grade 2 (9) & $3(33.3 \%)$ & $6(66.7 \%)$ & \multirow{3}{*}{$\mathrm{P}=0.378$} \\
\hline & Grade 3 (9) & $5(55.6 \%)$ & $4(44.4 \%)$ & \\
\hline & Grade 4 (7) & $5(71.4 \%)$ & $2(28.6 \%)$ & \\
\hline & Total & $\mathrm{N}=13(52 \%)$ & $\mathrm{N}=12(48 \%)$ & \\
\hline
\end{tabular}

Relation between preoperative DAs and intra-operative tumor consistency:

No statistical differences were found as regards the intraoperative tumor consistency in relation to the preoperative hormonal treatment status of prolactinoma patients. In group 1 patients only 3 cases out of $12(25 \%)$ were found to have firm tumor in consistency during intraoperative excision in contrast to 3 out of $13(23.1 \%)$ in group 2 patients with insignificant $\mathrm{p}$ value $=1.000$ (chart $1 \&$ table 2$)$.

Chart 1: Hormonal treatment in relation to intra-operative consistency

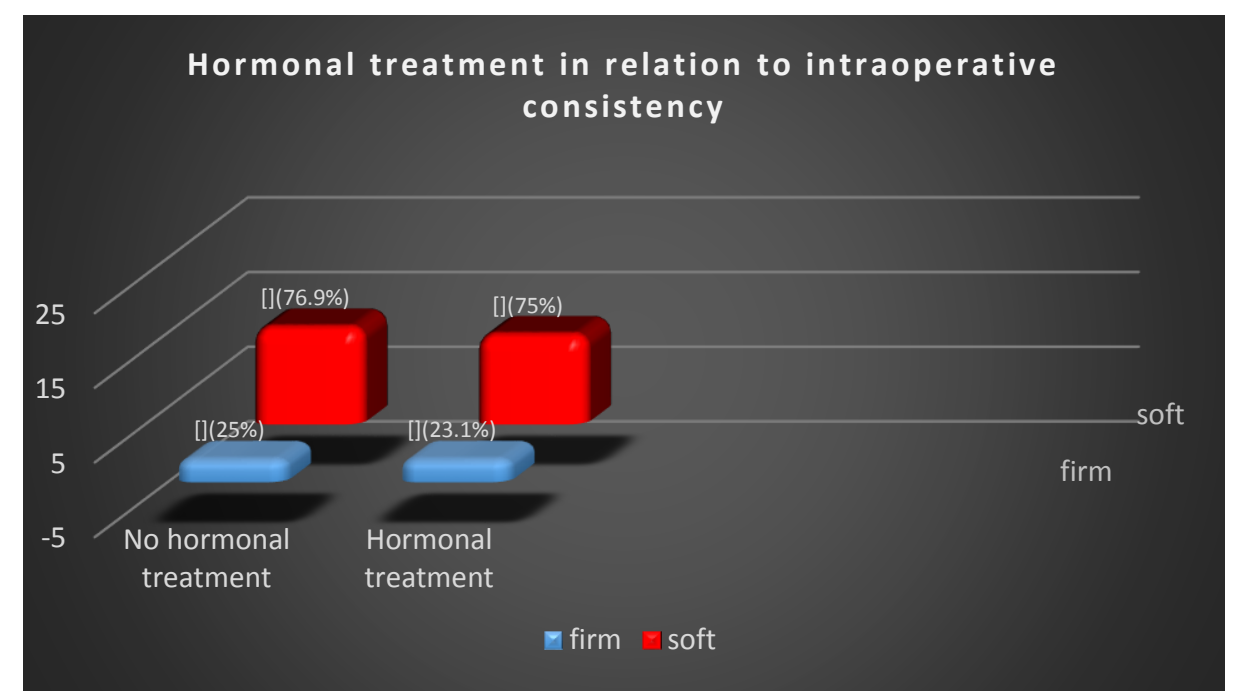

Table 2: Hormonal treatment in relation to intra-operative consistency

\begin{tabular}{|c|c|c|c|c|}
\hline \multirow{4}{*}{ Intraoperative consistency } & Group 1 & Group 2 & P value \\
\cline { 2 - 5 } & Soft & $9(75 \%)$ & $10(76.9 \%)$ & \multirow{2}{*}{ P $=1.000$} \\
\cline { 2 - 5 } & Firm & $3(25 \%)$ & $3(23.1 \%)$ & \\
\cline { 2 - 5 } & & 12 & 13 & \\
\hline
\end{tabular}

Extent of surgical excision in relation to hormonal treatment and knosp classification

We didn't find significant statistical difference as regards the extent of surgical excision between the hormonal and non-hormonal receiving groups. Growth total removal was done in $58.3 \%$ in Group 1 in contrast to $38.5 \%$ of Group 2. Incomplete tumor excision, Subtotal removal and partial removal, was achieved in $41.7 \%$ of Group 1 versus $61.5 \%$ in Group 2 with insignificant $\mathrm{p}$ value $=0.320$ (table 3).

The extent of surgical excision was found inversely proportional to the tumor Knosp classification. Highly significant statistical difference was found when we compared the tumor 
grade by Knosp classification versus the extent of surgical removal achieved. One hundred percent GTR was achieved in all Knosp grade 2 prolactinoma patients (ratio $=9 / 9$ ) in comparison to only $18.75 \%$ of KNOSP grade $3 \&$ grade 4 patients (ratio=3/16) which give highly significant $\mathrm{p}$-value ( $100 \%$ GTR vs $18.75 \%$ NTR\&PR; $p=<0.001$ ) (table 3 \&chart 2).

Table 3: Extent of removal in relation to knosp grade and Hormonal treatment

\begin{tabular}{|c|c|c|c|c|c|c|}
\hline & & $\begin{array}{c}\text { Knosp grade 2 } \\
(9)\end{array}$ & $\begin{array}{c}\text { Knosp grade 3\&4 } \\
(16)\end{array}$ & $\begin{array}{c}\text { Group } \\
1\end{array}$ & $\begin{array}{c}\text { Group } \\
2\end{array}$ & P value \\
\hline \multirow{2}{*}{$\begin{array}{c}\text { Extent of surgical } \\
\text { removal }\end{array}$} & GTR & 9 & 3 & $7(58.3 \%)$ & $5(38.5 \%)$ & P $=0.320$ \\
\cline { 2 - 7 } & STR+PR & 0 & 13 & $5(41.7 \%)$ & $8(61.5 \%)$ & \\
\hline
\end{tabular}

GTR; growth total removal , STR; subtotal removal , PR; partial removal

Chart 2: Knosp grade in relation to extent of surgical removal

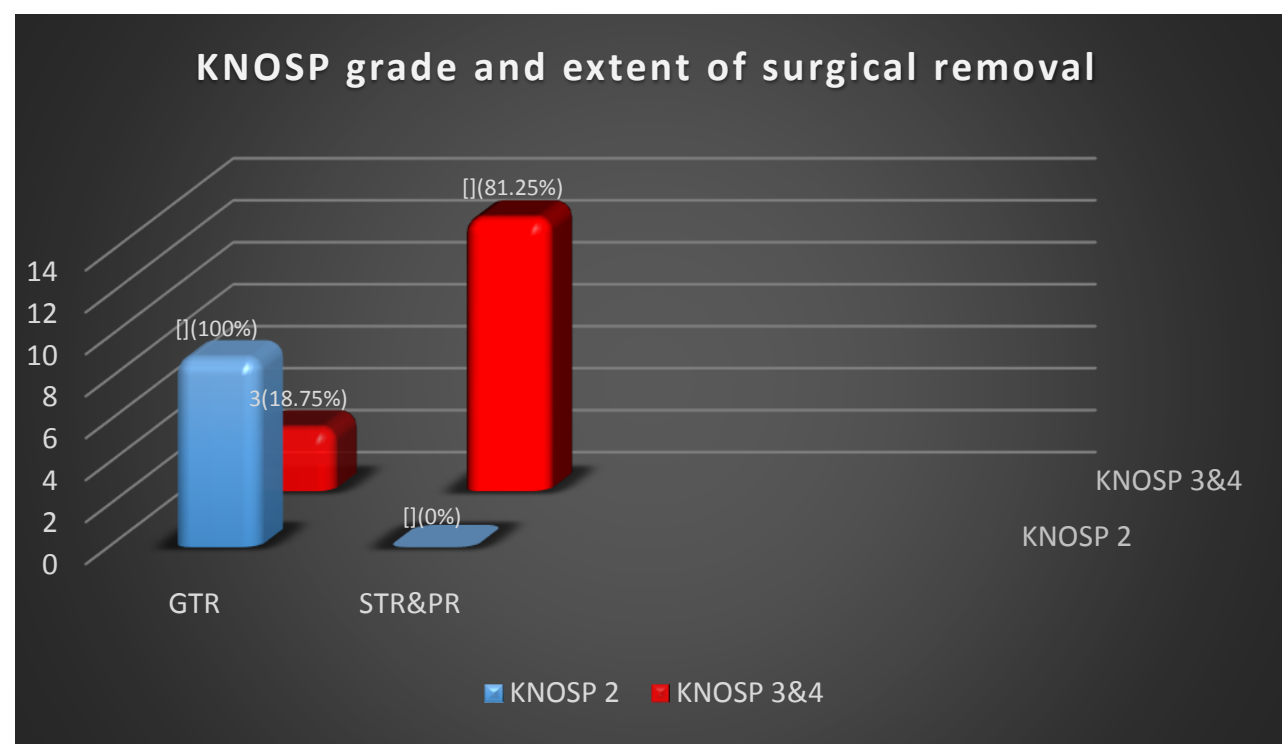

GTR; growth total removal, STR; subtotal removal, PR; partial removal

Effect of surgical excision extent on post-operative hormonal remission

Extent of surgical excision was positively reflected on post-operative prolactin level with nearly significant difference. Of the GTR group 11 patient out of $12(91.7 \%)$ had normal post-operative prolactin level 3 months after surgery with only one patient $(8.3 \%)$ returned with high postoperative serum prolactin levels that required treatment $(91.7 \%$ GTR remission vs $8.3 \% ; \mathrm{p}=0.073)$. In the other group of STR\&PR only nearly half of the patients $(53.8 \%)$ had normal post-operative prolactine while the remaining patients $(46.2 \%)$ were still suffering from high post-operative prolactin level that required further management (table 4 \&chart 3 ).

Table 4: Extent of surgical removal in relation to post-operative prolactin level

\begin{tabular}{|c|c|c|c|}
\hline \multirow{2}{*}{ Postoperative prolactin } & \multicolumn{2}{|c|}{$\begin{array}{c}\text { Extent of surgical } \\
\text { removal }\end{array}$} & \multirow{2}{*}{ P value } \\
\cline { 2 - 3 } & GTR & STR+PR & \multirow{2}{*}{$\mathrm{P}=0.073$} \\
\hline Normal & $11(91.7 \%)$ & $7(53.8 \%)$ & \multirow{2}{*}{$6(46.2 \%)$} \\
\hline High & $1(8.3 \%)$ & & \\
\hline
\end{tabular}

GTR; growth total removal, STR; subtotal removal, PR; partial removal 
Chart 3: Extent of surgical removal in relation to post-operative prolactin level

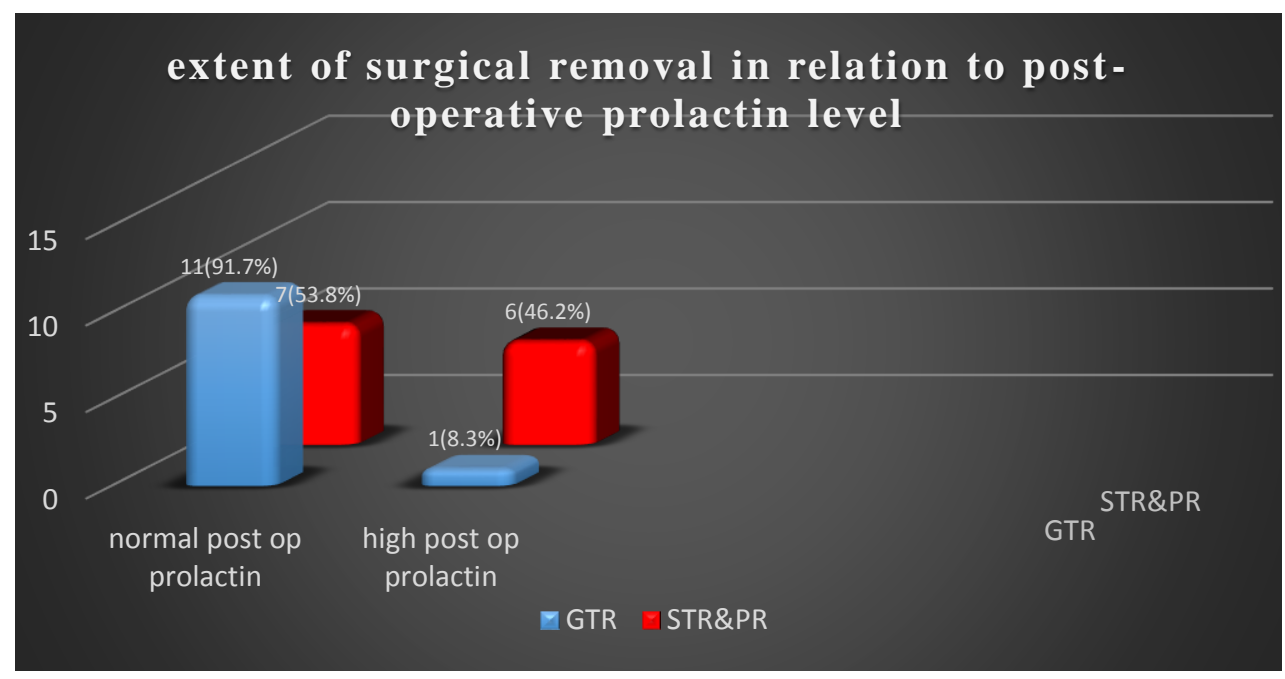

GTR; growth total removal, STR; subtotal removal, PR; partial removal

\section{Discussion:}

Several reports suggested that DA medications are accused for prolactinoma fibrosis $(\mathbf{2 , 5 , 1 0 )}$. but whether fibrosis has a positive or a negative influence on the management of prolactinomas is still of debate. In our study we observed no significant difference in the frequency or extent of fibrosis between patients regardless of their preoperative hormonal therapy. No statistical differences were found as regards the intraoperative tumor consistency in relation to the preoperative hormonal treatment status of prolactinoma patients. the intraoperative nature of the usually soft suckable prolactinoma were found in $76 \%$ of our cases regardless of the hormonal therapy.

While some neurosurgeons refer that harder tumors may be more difficult to resect, and this may increase the risk of intraoperative and postoperative complications. in our study the preoperative hormonal therapy did not affect the extent of gross surgical resection achieved.

In a study at Johns Hopkins Hospital between 1992 and 2009. there was no difference between "fibrous" and "non fibrous" adenomas in intra-operative or post-operative complications (17). The same results appear in our study as we didn't face any cases with major intraoperative vascular bleeding, and we didn't need to do any extracapsular work in both groups.

Regarding the post-operative complications, we did not find any difference between the two groups with post-operative vision improvement or at least the same regardless of the hormonal treatment and no major morbidity or mortality in the whole series.

In this study the extent of surgical excision was found inversely proportional to the tumor Knosp classification. Highly significant statistical difference was found when we compared the tumor grade by KNOSP classification and the extent of surgical removal achieved. Also we found that the extent of surgical excision was positively reflected on post-operative hormonal remission of prolactin. These results are similar to the Chinese study conducted by Han, Yan-Long and others in 2018 study who achieve the highest remission rate in microadenomas for which GTR were $100 \%$ followed by macroadenoma which also showed resection percentage higher than the giant ones ${ }^{(18)}$.

\section{Conclusions:}

In conclusion, the results of the current study are in agreement with studies using dopamine agonist as the primary treatment for prolactinoma. Surgical excision is deferred for failure or intolerance of side effects of the the hormonal therapy. There is no risk of more tumor fibrosis nor increase in the complication rate due to DA medication prior to surgery. Our results are not consistent with the hypothesis that more fibrosis occurs and complications increase after hormonal therapy. So, we recommend not to rush for surgery for cases of prolactinoma from the start without giving the patient the chance of cure using DA therapy for at least 3 months.

It was found that the only factor that affect the post-operative hormonal remission is the extent of surgical excision. So, we recommend that we 
should do our best and use all the available resources to excise as much as we can from the tumor aiming gross total resection.

\section{References}

1.Gillam MP, Molitch ME, Lombardi G, Colao A (2006): Advances in the treatment of prolactinomas. Endocrine reviews, 27(5):485534

2.Bevan JS, Webster J, Burke CW, Scanlon MF (1992): Dopamine agonists and pituitary tumor shrinkage. Endocrine reviews, 13(2):220-40

3.Tyrrell JB, Lamborn KR, Hannegan LT, Applebury CB, Wilson CB (1999): Transsphenoidal microsurgical therapy of prolactinomas: initial outcomes and long-term results. Neurosurgery, 44(2):254-61

4.Gen M, Uozumi T, Ohta M, Ito A, Kajiwara H, Mori S (1984): Necrotic changes in prolactinomas after long term administration of bromocriptine. The Journal of Clinical Endocrinology \& Metabolism, 59(3):463-70

5.Landolt AM, Osterwalder V (1984): Perivascular fibrosis in prolactinomas: is it increased by bromocriptine. The Journal of Clinical Endocrinology \& Metabolism, 58(6):1179-83

6.Esiri M, Bevan J, Burke C, Adams C (1986): Effect of bromocriptine treatment on the fibrous tissue content of prolactin-secreting and nonfunctioning macroadenomas of the pituitary gland. The Journal of Clinical Endocrinology \& Metabolism, 63(2):383-8

7.Takahashi T, Kuwayama A, Katoh T, Kageyama N (1986): Histological changes and operative findings of pituitary adenomas after bromocriptine treatment. Nihon Naibunpi Gakkai Zasshi, 62(12):1336-51

8.Hubbard JL, Scheithauer BW, Abboud CF, Laws ER (1987): Prolactin-secreting adenomas: the preoperative response to bromocriptine treatment and surgical outcome.Journal of neurosurgery, 67(6):81621

9.Scanarini M (1990): Morphological changes in prolactinoma induced by bromocriptine treatment.Minerva endocrinologica, 15(1):135
10.Nishioka H, Haraoka J, Akada K, Azuma S (2002): Gender-related differences in prolactin secretion in pituitary prolactinomas. Neuroradiology, 44(5):407-10

11.Molitch ME, Thorner MO, Wilson C, Molitch ME, Thorner MO, Wilson C (1997): The case for initial surgical removal of certain prolactinomas. The Journal of Clinical Endocrinology \& Metabolism, 82(4):9961000

12.Losa M, Mortini P, Barzaghi R, Gioia L, Giovanelli M (2002): Surgical treatment of prolactin-secreting pituitary adenomas: early results and long-term outcome. The Journal of Clinical Endocrinology \& Metabolism, 87(7):3180-6

13.Hamilton DK, Vance ML, Boulos PT, Laws ER (2005): Surgical outcomes in hyporesponsive prolactinomas: analysis of patients with resistance or intolerance to dopamine agonists. Pituitary, 8(1):53-60

14.Xu Z, Su C, Ren Z, Wang R, Yang Y, Ma W (2008): Transsphenoidal microsurgical results of non-invasive prolactinomas. Zhonghua wai ke za zhi [Chinese journal of surgery], 46(4):293-5

15.Saeger W, Mohr K, Caselitz J, Lüdecke D (1986): Light and electron microscopical morphometry of pituitary adenomas in hyperprolactinemia. Pathology-Research and Practice, 181(5):544-50

16.Bălinişteanu $B$, Ceauşu R, Cîmpean AM, Baciu I, Băculescu N, Coculescu M (2011): Conventional examination versus immunohistochemistry in the prediction of hormone profile of pituitary adenomas. An analysis on 142 cases. Romanian journal of morphology and embryology, 52(3):1041-5

17.Menucci M, Quiñones-Hinojosa A, Burger P, Salvatori R (2011): Effect of dopaminergic drug treatment on surgical findings in prolactinomas. Pituitary , 14(1):68-74

18.Han Y, Chen D, Zhang C, Pan M, Yang X, Wu $Y$ (2018): Retrospective analysis of 52 patients with prolactinomas following endoscopic endonasal transsphenoidal surgery. Medicine , 97(45) 